\title{
Complex Projective Synchronization in Drive-Response Stochastic Complex Networks by Impulsive Pinning Control
}

\author{
Xuefei Wu and Zhe Nie \\ School of Computer and Software Engineering, Shenzhen Polytechnic, Shenzhen 518055, China \\ Correspondence should be addressed to Zhe Nie; niezhe@szpt.edu.cn
}

Received 18 November 2013; Accepted 27 January 2014; Published 5 March 2014

Academic Editor: Jagannathan Sarangapani

Copyright ( $) 2014$ X. Wu and Z. Nie. This is an open access article distributed under the Creative Commons Attribution License, which permits unrestricted use, distribution, and reproduction in any medium, provided the original work is properly cited.

\begin{abstract}
The complex projective synchronization in drive-response stochastic coupled networks with complex-variable systems is considered. The impulsive pinning control scheme is adopted to achieve complex projective synchronization and several simple and practical sufficient conditions are obtained in a general drive-response network. In addition, the adaptive feedback algorithms are proposed to adjust the control strength. Several numerical simulations are provided to show the effectiveness and feasibility of the proposed methods.
\end{abstract}

\section{Introduction}

Complex networks become more and more important in modern society. Up to now, the investigation on the synchronization of complex networks has attracted a great deal of attentions due to its potential applications in various fields, such as physics, secure communication, automatic control, biology, and sociology [1-5]. In literature, there are many widely studied synchronization patterns, which define the correlated in-time behaviors among the nodes in a dynamical network, for example, complete synchronization $[6-8]$, phase synchronization $[9,10]$, lag synchronization [1113], antisynchronization [14-16], projective synchronization [17-29], and so on. Projective synchronization reflects a kind of proportionality between the synchronized states, so it is an interesting research topic and has many applications.

Recently, projective synchronization under various cases of complex dynamical networks has been studied [1729]. In [19], Du et al. studied the problem of function projective synchronization for general complex dynamical networks with time delay. A hybrid feedback control method is designed to achieve function projective synchronization for complex dynamical networks with constant time or time-varying delay. In [20], Liu investigated the synchronization problem of fractional-order complex networks with nonidentical nodes, and the generalized projective synchronization criterion of fractional-order complex networks with order $0<q<1$ is obtained. In [21], Yao and Wang explored a new cluster projective synchronization scheme in time-varying delay coupled complex dynamical networks with nonidentical nodes in consideration of the community structure of the networks.

In most existing research, the two complex networks (socalled driver-response networks) evolve along the same or inverse direction with respect to real number, real matrix, or even real function in a complex plane $[17,19-21,23-$ 29]. However, in real world, the systems can often evolve in different directions with a constant intersection angle with respect to complex number; for example, $y=\rho e^{j \theta} x$, where $x$ denotes the drive system, $y$ denotes the response system, $\rho>0$ denotes the zoom rate, $\theta \in[0,2 \pi)$ denotes the rotation angle, and $j=\sqrt{-1}$. This synchronization scheme has a large number of real-life examples. For instance, in distributed computers collaboration, each distributed computer (response system) not only to receive unified command from server (drive system), but also they are mutual to use resources for collaboration [30]. Furthermore, in a social network or games in economic activities, and behaviors of individuals (those response systems) will be affected not only by powerful one (the drive system used in the present paper), 
but also those with a similar role as themselves [22]. Recently, some related works have come out, such as $[18,22]$. In $[18$, 22 ], $\mathrm{Wu}$ et al. introduced the concept of complex projective synchronization based on Lyapunov stability theory, several typical chaotic complex dynamical systems are considered and the corresponding controllers are designed to achieve the complex projective synchronization.

In many systems, the impulsive effects are common phenomena due to instantaneous perturbations at certain moments [31-33]. In the past several years, impulsive control strategies have been widely used to stabilize and synchronize coupled complex dynamical system, such as signal processing system, computer networks, automatic control systems, and telecommunications. In [31], Cai et al. investigated the robust impulsive synchronization of complex delayed dynamical networks. Yang and Cao [32] studied the exponential synchronization of complex dynamical network with a coupling delay and impulsive disturbance. Zhu et al. gave some global impulsive exponential synchronization criteria of time-delayed coupled chaotic systems in [33]. Xu et al. studied the synchronization problem of stochastic complex networks with Markovian switching and time-varying delays are investigated by using impulsive pinning control scheme in [34].

Besides, due to the finite information transmission and processing speeds among the units, the connection delays in realistic modeling of many large networks with communication must be taken into account, such as [19, 23, 24, 35]. What is more, uncertainties commonly exist in the real world, such as stochastic forces on the physical systems and noisy measurements caused by environmental uncertainties; the stochastic forms from the same noise of one-dimensional vector to the different noise perturbations of vector were investigated in $[9,10,15,17,25]$. Therefore, it is important to study the effect of time delay and stochastic noise in complex projective synchronization of drive-response networks.

Based on the above-mentioned content, the complex projective synchronization in drive-response stochastic coupled networks with complex-variable systems and linear coupling time delays by impulsive pinning control scheme are considered in this paper. Several simple and practical criteria for complex projective synchronization are obtained by using Lyapunov functional method, stochastic differential theory, and linear matrix inequality (LMI) approaches.

Notation. Throughout this paper, $\mathbb{C}^{n}$ and $\mathbb{C}^{m \times n}$ denote $n$ dimensional complex vectors and the set of $m \times n$ complex matrices, respectively. For Hermite matrix $H$, the notation $H>0(H<0)$ means that the matrix $H$ is positive definite (negative definite). For any complex (real) matrix $M$, $M^{s}=M^{T}+\bar{M}$. For any complex number (or complex vector) $x$, the notations $x^{r}$ and $x^{i}$ denote its real and imaginary parts, respectively, and $\bar{x}$ denotes the complex conjugate of $x$. $\lambda_{\text {min }}(A)\left(\lambda_{\text {max }}(A)\right)$ represents the smallest(largest) eigenvalue of a symmetric matrix $A$. $\otimes$ is the Kronecker product. The superscript $T$ of $x^{T}$ or $A^{T}$ denotes the transpose of the vector $x \in \mathbb{R}^{n}$ or the matrix $A \in \mathbb{R}^{m \times n}$. $I_{n}$ is identity matrix with $n$ nodes.

\section{Model Description and Preliminaries}

Consider a drive-response network coupled with $1+N$ identical partially linear stochastic complex network with coupling time delay, which is described as follows:

$$
\begin{gathered}
\dot{x}(t)=M(z(t)) x(t) \\
\dot{z}(t)=f(x(t), z(t)) \\
d y_{i}(t)=\left\{M(z(t)) y_{i}(t)+\varepsilon_{1} \sum_{k=1}^{N} a_{i k} \Gamma y_{k}(t)\right. \\
\left.+\varepsilon_{2} \sum_{k=1}^{N} b_{i k} \Gamma y_{k}(t-\tau)\right\} d t \\
+\sigma_{i}(y(t), y(t-\tau)) d w_{i}(t), \quad i=1,2, \ldots, N
\end{gathered}
$$

where $x(t)=\left(x_{1}, x_{2}, \ldots, x_{m}\right)^{T} \in \mathbb{C}^{m}$ and $z(t) \in \mathbb{R}$ are the drive system variables and $y_{i}(t)=\left(y_{i 1}, y_{i 2}, \ldots, y_{i m}\right)^{T} \in \mathbb{C}^{m}$ is the state variable of a node $i$ in the response network. $M(z(t)) \in \mathbb{R}^{m \times m}$ is a complex matrix function, $\varepsilon_{1}>0, \varepsilon_{2}>$ 0 is the coupling strength, and $\Gamma \in \mathbb{R}^{m \times m}$ is the inner coupling matrix. $\tau$ is the coupling time delay. Matrices $A=$ $\left(a_{i j}\right)_{N \times N}$ and $B=\left(b_{i j}\right)_{N \times N}$ are the zero-row-sum outer coupling matrix, which denote the network topology and are defined as follows. If there is a connection (information transmission) from node $j$ to node $i(i \neq j)$, then $a_{i j} \neq 0$ and $b_{i j} \neq 0$; otherwise, $a_{i j}=0$ and $b_{i j}=0$, and $w_{i}(t)=\left(w_{i 1}(t)\right.$, $\left.w_{i 2}(t), \ldots, w_{i n}(t)\right)^{T} \in \mathbb{R}^{n}$ is a bounded vector-form Weiner process, satisfying

$$
\begin{aligned}
& \mathbb{E} w_{i j}(t)=0, \quad \mathbb{E} w_{i j}^{2}(t)=1, \\
& \mathbb{E} w_{i j}(t) w_{i j}(s)=0 \quad(s \neq t) .
\end{aligned}
$$

Now, two mathematical definitions for the generalized projective synchronization are introduced as follows.

Definition 1. If there is a complex $\alpha$ such that

$$
\sum_{i=1}^{N} \mathbb{E}\left\|y_{i}(t)-\alpha x_{i}(t)\right\|^{2} \leq K e^{-\kappa t},
$$

for some $K>0$ and some $\kappa>0$, then the drive-response networks (1) and (2) are said to achieve complex projective synchronization in mean square, and the parameter $\alpha$ is called a scaling factor.

Without loss of generality, let $\alpha=\rho(\cos \theta+j \sin \theta)$, where $\rho=|\alpha|$ is the module of $\alpha$ and $\theta \in[0,2 \pi)$ is the phase of $\alpha$. Therefore, the projective synchronization is achieved when $\theta=0$ or $\pi$. Furthermore, the complete synchronization is achieved when $\rho=1$ and $\theta=0$, and the antisynchronization is achieved when $\rho=1$ and $\theta=\pi$ [22]. 
Definition 2 (see [22]). Matrix $A=\left(a_{i k}\right)_{i, k}^{N}$ is said to belong to class $A_{1}$, denoted as $A \in A_{1}$, if

(1) $a_{i k} \geq 0, i \neq k, a_{i i}=-\sum_{k=1, k \neq i}^{N} a_{i k}=-\sum_{k=1, k \neq i}^{N} a_{k i}, i=$ $1,2, \ldots, N$

(2) $A$ is irreducible.

The following lemmas and assumption are used throughout the paper.

Lemma 3 (see [22]). Let $m \times m$ complex matrix $H$ be Hermitian; then

(1) $x^{T} H \bar{x}$ is real for all $x \in C^{m}$;

(2) all the eigenvalues of $H$ are real.

Lemma 4 (see [35]). If $A=\left(a_{i j}\right)_{m \times m}$ is irreducible, $a_{i j}=a_{j i} \geq$ 0 , for $i \neq j$, and $\sum_{j=1}^{m} a_{i j}=0$, for all $i=1,2, \ldots, m$, then all eigenvalues of the matrix

$$
\left(\begin{array}{cccc}
a_{11}-\epsilon & a_{12} & \cdots & a_{1 m} \\
a_{21} & a_{22} & \cdots & a_{2 m} \\
\vdots & \vdots & \ddots & \vdots \\
a_{m 1} & a_{m 2} & \cdots & a_{m m}
\end{array}\right)
$$

are negative for any positive constant $\epsilon$.

Lemma 5 (see [30, 36]). Consider an n-dimensional stochastic differential equation

$$
\begin{aligned}
d x(t)= & f(t, x(t), x(t-\tau)) d t \\
& +\sigma(t, x(t), x(t-\tau)) d \omega(t) .
\end{aligned}
$$

Let $C^{2,1}\left(\mathbb{C}_{+} \times \mathbb{C}^{n} ; \mathbb{R}_{+}\right)$denote the family of all nonnegative functions $V(t, x)$ on $\mathbb{R}_{+} \times \mathbb{C}^{n}$, which are twice continuously differentiable in $x$ and once differentiable in $t$. If $V \in C^{2,1}\left(\mathbb{R}_{+} \times\right.$ $\left.\mathbb{C}^{n} ; \mathbb{R}_{+}\right)$, define an operator $\mathscr{L} V$ from $\mathbb{R}_{+} \times \mathbb{C}^{n}$ to $\mathbb{R}$ by

$$
\begin{aligned}
\mathscr{L} V(t, x)= & V_{t}(t, x)+V_{x}(t, x) f(t, x, y) \\
& +\frac{1}{2} \operatorname{Tr}\left[\sigma(t, x, y)^{T} V_{x x} \sigma(t, x, y)\right],
\end{aligned}
$$

where $V_{t}(t, x)=\partial V(t, x) / \partial t, V_{x}(t, x)=\left(\partial V(t, x) / \partial x_{1}, \ldots\right.$, $\left.\partial V(t, x) / \partial x_{n}\right)$, and $V_{x x}(t, x)=\left(\partial^{2} V(t, x) / \partial x_{i} x_{j}\right)_{n \times n}$. If $V \in$ $C^{2,1}\left(\mathbb{R}_{+} \times \mathbb{C}^{n} ; \mathbb{R}_{+}\right)$, then, for any $\infty>t>t_{0} \geq 0$,

$$
\mathbb{E} V(t, x(t))=\mathbb{E} V\left(t_{0}, x\left(t_{0}\right)\right)+\mathbb{E} \int_{t_{0}}^{t} \mathscr{L} V(s, x(s)) d s
$$

as long as the expectations of the integrals exist.

Assumption 6 (see [22]). Suppose that there exists a constant $L$ such that the largest eigenvalue of $M^{s}(z(t))$ satisfies

$$
\lambda_{\max }\left(M^{s}(z(t))\right) \leq L .
$$

Remark 7. All the chaotic systems satisfy Assumption 6 due to the fact that $z(t)$ is bounded [22].
Assumption 8. Denote $e_{i}(t)=y_{i}(t)-\alpha x(t)$, and suppose $\sigma_{i}(e(t), e(t-\tau))=\sigma_{i}(y(t), y(t-\tau))$. Then, there exist positive definite constant matrices $\Upsilon_{i 1}$ and $\Upsilon_{i 2}$, for $i=1,2, \ldots, N$, such that

$$
\begin{aligned}
& \operatorname{Tr}\left[\sigma_{i}^{T}(e(t), e(t-\tau)) \overline{\sigma_{i}(e(t), e(t-\tau))}\right] \\
& \quad \leq \sum_{j=1}^{N} e_{j}^{T}(t) \Upsilon_{i 1} \overline{e_{j}(t)}+\sum_{j=1}^{N} e_{j}^{T}(t-\tau) \Upsilon_{i 2} \overline{e_{j}(t-\tau)} .
\end{aligned}
$$

Remark 9. Assumption 8 is easily satisfied; for instance, because of existing noise in the process of information transmission, the noise strength is $\sigma_{i}(y(t), y(t-$ $\tau))=\mid \bar{\sigma}_{i} \sum_{k=1}^{N} a_{i k}\left(y_{k}(t)-y_{i}(t)\right)+\widetilde{\sigma}_{i} \sum_{k=1}^{N} b_{i k}\left(y_{k}(t-\tau)-y_{i}(t-\right.$ $\tau)) \mid$, which depends on the states of the nodes, where $\bar{\sigma}_{i}$ and $\tilde{\sigma}_{i}$ are constants, $i=1,2, \ldots, N$, so that $\Upsilon_{i 1}=\bar{\sigma}_{i} N$ diag $\left\{a_{i 1}^{2}, a_{i 2}^{2}, \ldots, a_{i N}^{2}\right\}, \Upsilon_{i 2}=\widetilde{\sigma}_{i} N \operatorname{diag}\left\{a_{i 1}^{2}, a_{i 2}^{2}, \ldots, a_{i N}^{2}\right\}$.

\section{Main Results}

Our objective here is to achieve complex projective synchronization in the drive-response networks (1) and (2) by adopting different control schemes. Firstly, several sufficient conditions are obtained for achieving complex projective synchronization in drive-response networks (1) and (2) by applying proper controllers $u_{i}(t)$ on the response network. Then, the controlled response network is

$$
\begin{aligned}
d y_{i}(t)= & \left\{M(z(t)) y_{i}(t)+\varepsilon_{1} \sum_{k=1}^{N} a_{i k} \Gamma y_{k}(t)\right. \\
& \left.+\varepsilon_{2} \sum_{k=1}^{N} b_{i k} \Gamma y_{k}(t-\tau)+u_{i}(t)\right\} d t \\
& +\sigma_{i}(y(t), y(t-\tau)) d w_{i}(t), \quad i=1,2, \ldots, N .
\end{aligned}
$$

Define the synchronization errors between the drive network (1) and the response network (2) as $e_{i}(t)=y_{i}(t)-$ $\alpha x(t)$, because of $\sigma_{i}(e(t), e(t-\tau))=\sigma_{i}(y(t), y(t-\tau))$; then we have the following error system:

$$
\begin{aligned}
d e_{i}(t)= & \left\{M(z(t)) e_{i}(t)+\varepsilon_{1} \sum_{k=1}^{N} a_{i k} \Gamma e_{k}(t)\right. \\
& \left.+\varepsilon_{2} \sum_{k=1}^{N} b_{i k} \Gamma e_{k}(t-\tau)+u_{i}(t)\right\} d t \\
& +\sigma_{i}(e(t), e(t-\tau)) d w_{i}(t), \quad i=1,2, \ldots, N .
\end{aligned}
$$


Next, we consider complex projective synchronization between (1) and (6) via impulsive control under the assumptions $A \in A_{1}, B \in A_{1}$, and $\Gamma>0$,

$$
\begin{gathered}
d e_{i}(t)=\left\{M(z(t)) e_{i}(t)+\varepsilon_{1} \sum_{k=1}^{N} a_{i k} \Gamma e_{k}(t)\right. \\
\left.+\varepsilon_{2} \sum_{k=1}^{N} b_{i k} \Gamma e_{k}(t-\tau)\right\} d t \\
+\sigma_{i}(t, e(t), e(t-\tau)) d w_{i}(t), \\
t \neq t_{k}, \quad k \in Z^{+}, \quad i=1,2, \ldots, N, \\
\Delta e_{i}\left(t_{k}\right)=\epsilon_{i k} e_{i}\left(t_{k}^{-}\right), \quad t=t_{k}, \quad k \in Z^{+}, i=1,2, \ldots, N .
\end{gathered}
$$

Theorem 10. Supposing that Assumptions 6 and 8 hold, $A \in$ $A_{1}, B \in A_{1}$, and $\Gamma>0$. The complex projective synchronization in the drive-response networks (1) and (2) with the impulsive control (14) can be achieved if the following condition is satisfied:

$$
\left(\gamma+a+b e^{\gamma \tau}\right)(\tau+T)+2 \ln \left|1+\epsilon_{v}\right|-\gamma T<0,
$$

where $\gamma>0, a=\lambda_{\max }\left(L I_{N m}+\varepsilon_{1} A^{s} \otimes \Gamma+\Upsilon_{i 1} \otimes I_{N}\right)$ and $b=\lambda_{\max }\left(\varepsilon_{2}(B \otimes \Gamma)^{s}+\Upsilon_{i 2} \otimes I_{N}\right)$.

Proof. Consider the Lyapunov functional candidate

$$
V(t)=\sum_{i=1}^{N} e_{i}^{T}(t) \overline{e_{i}(t)}
$$

For any $t \in\left[t_{k-1}, t_{k}\right), k=1,2, \ldots$, by Lemma 5 , we have

$$
\begin{gathered}
\mathscr{L} V(t)=\sum_{i=1}^{N}\left\{\left[e_{i}^{T}(t) M^{T}(z(t))+\varepsilon_{1} \sum_{k=1}^{N} a_{i k} e_{k}^{T}(t) \Gamma^{T}\right.\right. \\
\left.\quad+\varepsilon_{2} \sum_{k=1}^{N} b_{i k} e_{k}^{T}(t-\tau) \Gamma^{T}\right] \overline{e_{i}(t)} \\
+e_{i}^{T}(t)\left[\overline{M(z(t)) e_{i}(t)}+\varepsilon_{1} \sum_{k=1}^{N} a_{i k} \overline{\Gamma e_{k}(t)}\right. \\
\left.\left.\quad+\varepsilon_{2} \sum_{k=1}^{N} b_{i k} \overline{e_{k}(t-\tau)}\right]\right\} \\
+\frac{1}{2} \sum_{i=1}^{N} \operatorname{Tr}\left[\sigma_{i}(e(t), e(t-\tau))^{T} \overline{\sigma_{i}(e(t), e(t-\tau))}\right] \\
\leq \sum_{i=1}^{N} L e_{i}^{T}(t) \overline{e_{i}(t)} \\
+\varepsilon_{1} \sum_{i=1}^{N} \sum_{k=1}^{N} a_{i k}\left[e_{k}^{T}(t) \Gamma^{T}+e_{i}^{T}(t) \Gamma\right] \overline{e_{k}(t)}
\end{gathered}
$$

$$
\begin{aligned}
& +\varepsilon_{2} \sum_{i=1}^{N} \sum_{k=1}^{N} b_{i k}\left[e_{k}^{T}(t-\tau) \Gamma^{T}+e_{i}^{T}(t) \Gamma\right] \overline{e_{k}(t-\tau)} \\
& +\sum_{i=1}^{N} e_{i}^{T}(t) \Upsilon_{i 1} \overline{e_{i}(t)}+\sum_{i=1}^{N} e_{i}^{T}(t-\tau) \Upsilon_{i 2} \overline{e_{i}(t-\tau)}
\end{aligned}
$$

Let $e(t)=\left(e_{1}^{T}, e_{2}^{T}, \ldots, e_{N}^{T}\right)$; then one has

$$
\begin{aligned}
\mathscr{L} V(t) \leq & e^{T}(t)\left(L I_{N m}+\varepsilon_{1} A^{s} \otimes \Gamma\right)+\Upsilon_{i 1} \overline{e(t)} \\
& +e^{T}(t-\tau)\left(\varepsilon_{2}(B \otimes \Gamma)^{s}+\Upsilon_{i 2}\right) \overline{e(t-\tau)} .
\end{aligned}
$$

In view of condition (14), we have

$$
\mathscr{L} V(t) \leq a V(t)+b V(t-\tau) .
$$

Define

$$
W(t)=e^{\gamma t} V(t)
$$

and use (14) to compute the operator

$$
\begin{aligned}
\mathscr{L} W(t) & =e^{\gamma t}[\gamma V(t)+\mathscr{L} V(t)] \\
& \leq e^{\gamma t}[\gamma V(t)+a V(t)+b V(t-\tau)],
\end{aligned}
$$

which, after applying the generalized Itô's formula, gives

$$
e^{\gamma t} \mathbb{E} V(t)=\mathbb{E} V(0)+\mathbb{E} \int_{0}^{t} \mathscr{L} W(s) d s
$$

for any $t_{k}>t>t_{0}>t_{k-1} \geq 0$. Hence, we have

$$
\begin{aligned}
e^{\gamma t} \mathbb{E} V(t) \leq & \mathbb{E} V\left(t_{0}\right) \\
& +\mathbb{E} \int_{t_{0}}^{t} e^{\gamma s}[\gamma V(s)+a V(s)+b V(s-\tau)] d s \\
\leq & \mathbb{E} V\left(t_{0}\right)+(\gamma+a) \int_{t_{0}}^{t} e^{\gamma s} \mathbb{E} V(s) d s \\
& +b e^{\gamma \tau} \int_{t_{0}}^{t} e^{\gamma(s-\tau)} \mathbb{E} V(s-\tau) d s .
\end{aligned}
$$

By changing variable $s-\tau=u$, we have

$$
\begin{aligned}
\int_{t_{0}}^{t} e^{\gamma(s-\tau)} \mathbb{E} V(s-\tau) d s & =\int_{t_{0}-\tau}^{t-\tau} e^{\gamma u} \mathbb{E} V(u) d u \\
& \leq \int_{t_{0}-\tau}^{t} e^{\gamma u} \mathbb{E} V(u) d u .
\end{aligned}
$$

Substituting (23) into (22), we get

$$
e^{\gamma t} \mathbb{E} V(t) \leq \mathbb{E} V\left(t_{0}\right)+\left(\gamma+a+b e^{\gamma \tau}\right) \int_{t_{0}-\tau}^{t} e^{\gamma u} \mathbb{E} V(u) d u .
$$




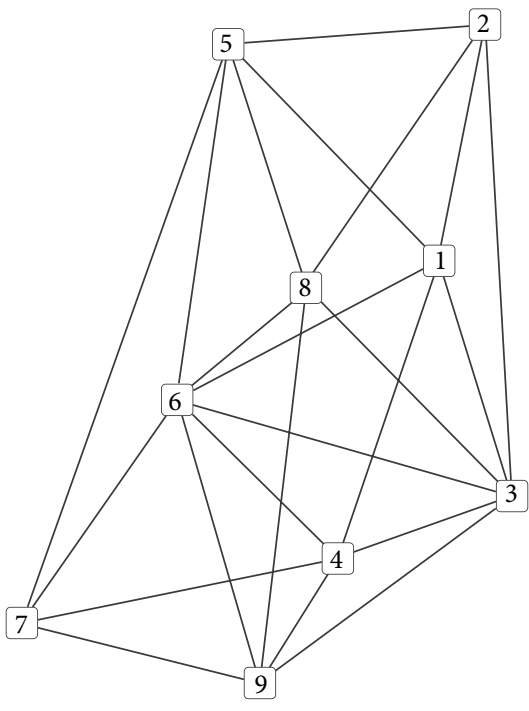

(a)

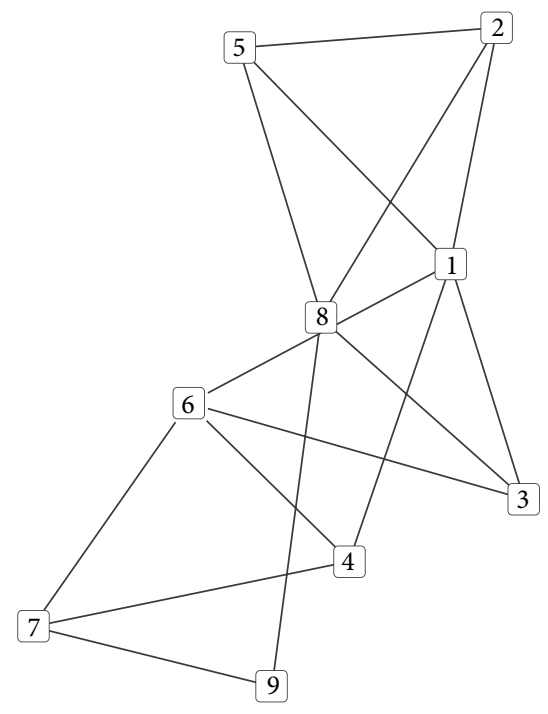

(b)

FIGURE 1: The topology structures of the networks for 9 nodes.

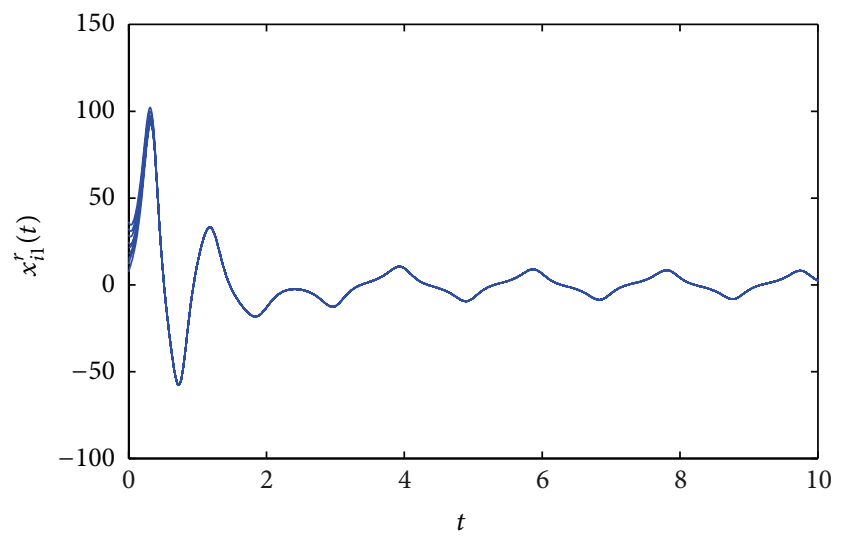

(a)

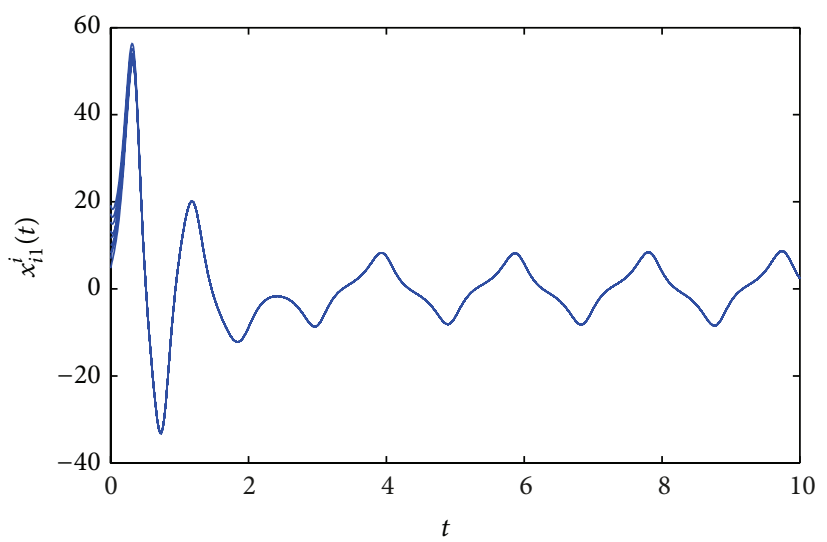

(c)

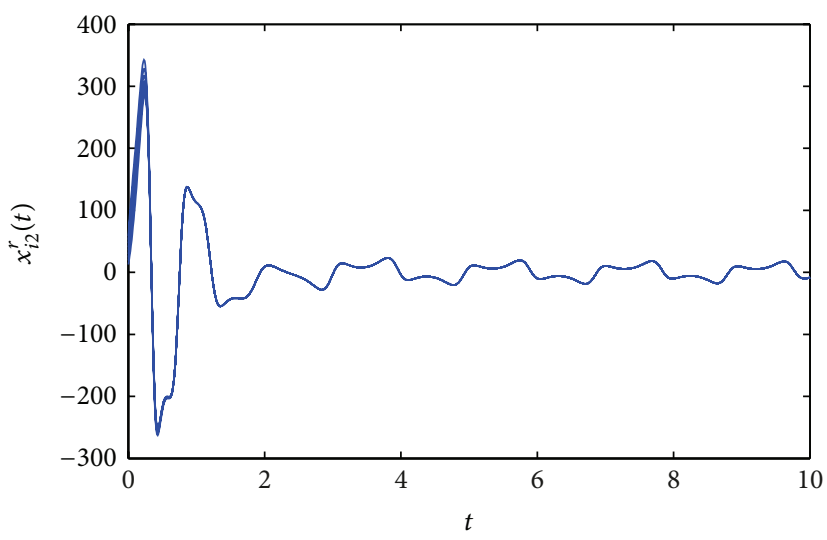

(b)

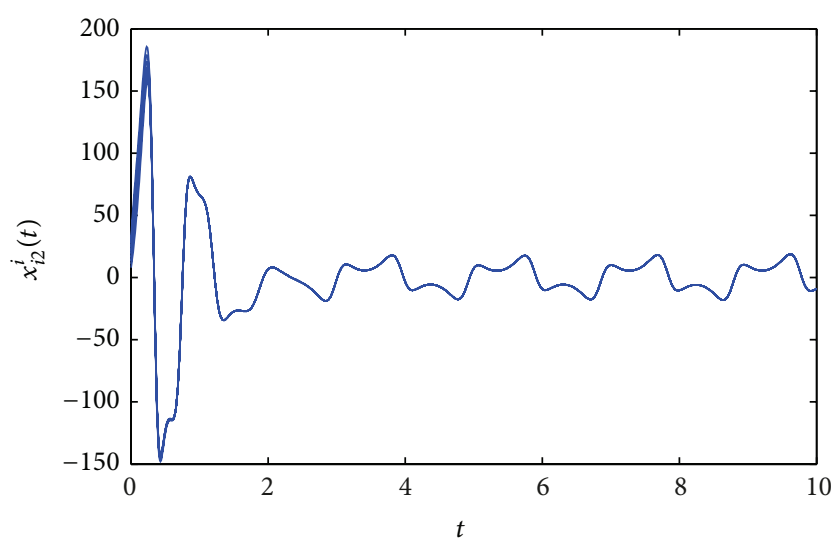

(d)

FIGURE 2: The evolution of the synchronization trajectory $y_{i}(i=1,2, \ldots, 10)$ by impulsive pinning control. 


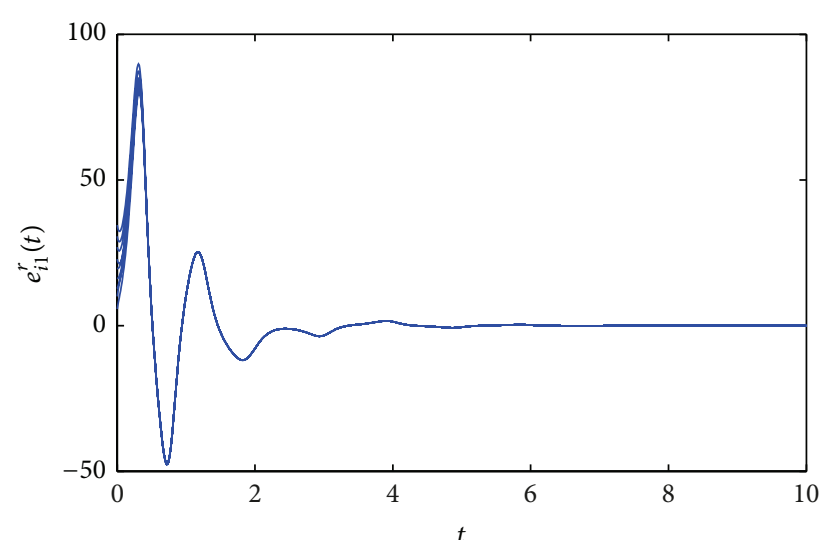

(a)

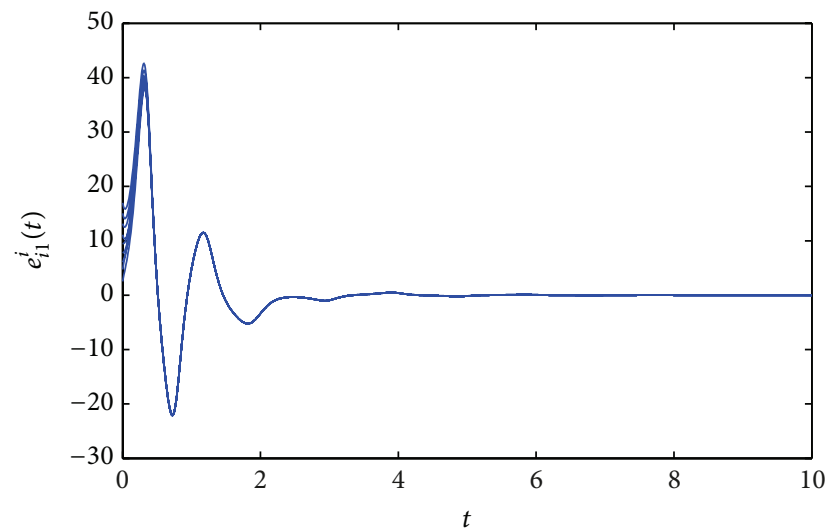

(c)

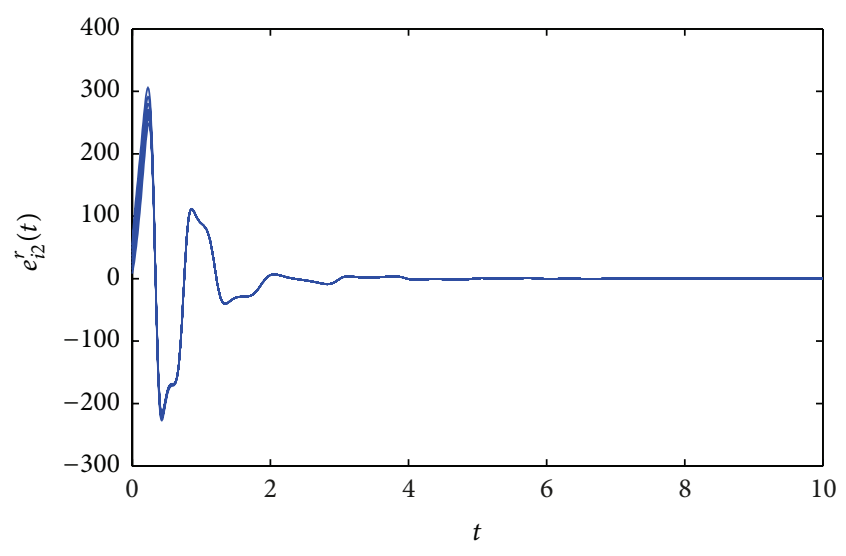

(b)

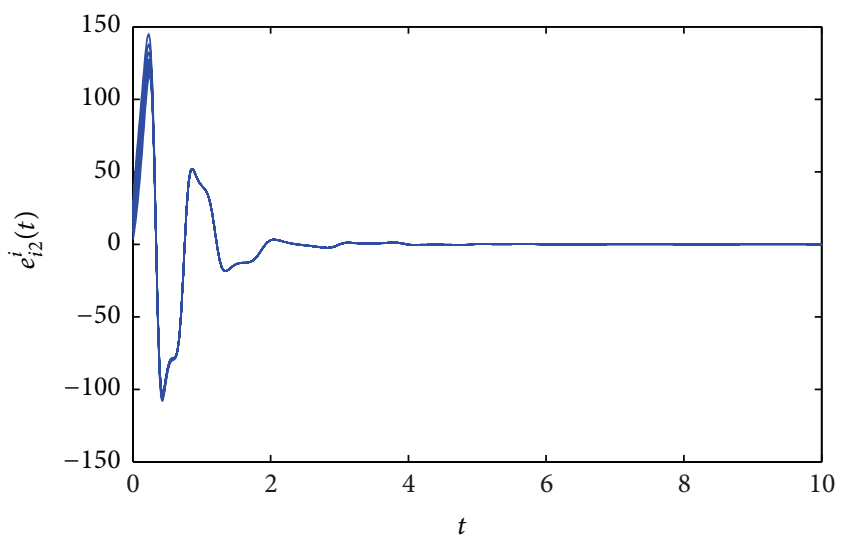

(d)

FIgURE 3: The evolution of the synchronization errors $y_{i}(i=1,2, \ldots, 10)$ by impulsive pinning control.

By using Gronwall's inequality, we get

$$
\mathbb{E} E(t) \leq \mathbb{E} E\left(t_{t_{0}}\right) e^{\left(\gamma+a+b e^{\gamma \tau}\right)\left(t-t_{t_{0}}+\tau\right)+\gamma\left(t_{0}-t\right)} .
$$

On the other hand, from the construction of $E(t)$, we have

$$
E\left(t_{k}\right) \leq\left(1+\epsilon_{k}\right)^{2} E\left(t_{k}^{-}\right)
$$

where $\left|1+\epsilon_{k}\right|=\max _{i=1,2, \ldots, N}\left|1+\epsilon_{i k}\right|$. According to (25) and (26), letting $k=\left\lfloor\left(t-t_{0}\right) / T\right\rfloor$, for any $t \in\left[t_{k-1}, t_{k}\right)$, we get

$$
\begin{aligned}
\mathbb{E} V(t) & \leq \mathbb{E} E\left(t_{k}\right) e^{\left(\gamma+a+b e^{\gamma \tau}\right)\left(t-t_{k}+\tau\right)+\gamma\left(t_{k}-t\right)} \\
& \leq \mathbb{E} V\left(t_{k-1}^{-}\right) e^{\left(\gamma+a+b e^{\gamma \tau}\right)\left(t-t_{k-1}+\tau\right)+\gamma\left(t_{k}-t\right)+2 \ln \left|1+\epsilon_{k-1}\right|} \\
& \leq \cdots \leq \mathbb{E} E(0) e^{\left(\gamma+a+b e^{\gamma \tau}\right)(t+k \tau)-\gamma t+\sum_{v=1}^{k-1} 2 \ln \left|1+\epsilon_{v}\right|}
\end{aligned}
$$

Letting $|1+\epsilon|=\max _{v \in Z^{+}}\left|1+\epsilon_{v}\right|$, we have

$$
\mathbb{E} E(t) \leq \mathbb{E} E(0) e^{\left(\gamma+a+b e^{\gamma \tau}\right)(t+k \tau)-\gamma t+2(k-1) \ln |1+\epsilon|} .
$$

Using condition (31) of Theorem 10, there exist positive numbers $K$ and $\kappa$ such that $\mathbb{E}\left\|e_{i}(t)\right\| \leq K e^{-\kappa\left(t-t_{0}\right)}$. The proof of Theorem 10 is completed.
When considering the system without delay, that is, $\tau=0$, we can derive the following controlled response network and the error system:

$$
\begin{aligned}
& d y_{i}(t)=\left\{M(z(t)) y_{i}(t)+\varepsilon_{1} \sum_{k=1}^{N} a_{i k} \Gamma y_{k}(t)+u_{i}(t)\right\} d t \\
& +\sigma_{i}(y(t)) d w_{i}(t), \quad i=1,2, \ldots, N, \\
& d e_{i}(t)=\left\{M(z(t)) e_{i}(t)+\varepsilon_{1} \sum_{k=1}^{N} a_{i k} \Gamma e_{k}(t)\right. \\
& \left.+\varepsilon_{2} \sum_{k=1}^{N} b_{i k} \Gamma e_{k}(t-\tau)\right\} d t \\
& +\sigma_{i}(t, e(t), e(t-\tau)) d w_{i}(t) \\
& t \neq t_{k}, \quad k \in Z^{+}, \quad i=1,2, \ldots, N, \\
& \Delta e_{i}\left(t_{k}\right)=\epsilon_{i k} e_{i}\left(t_{k}^{-}\right), \quad t=t_{k}, k \in Z^{+}, i=1,2, \ldots, N \text {. }
\end{aligned}
$$

Then, without loss of generality, one has the following corollary. 
Corollary 11. Supposing that Assumptions 6 and 8 hold, $A \in$ $A_{1}, B \in A_{1}$, and $\Gamma>0$. The complex projective synchronization in the drive-response networks (1) and (29) with the impulsive control (31) can be achieved if the following condition is satisfied:

$$
(\gamma+a)(\tau+T)+2 \ln \left|1+\epsilon_{v}\right|-\gamma T<0
$$

where $\gamma>0, a=\lambda_{\max }\left(L I_{N m}+\varepsilon_{1} A^{s} \otimes \Gamma+\Upsilon_{i 1} \otimes I_{N}\right)$.

\section{Numerical Simulations}

In this section, we conduct some numerical simulations to illustrate the effectiveness of the theorems of the previous section.

Consider a driver-response network coupled with the following complex Lorenz systems:

$$
\begin{gathered}
\dot{x}=M(z) x, \\
\dot{z}=-b z+\frac{1}{2}\left(\bar{x}_{1} x_{2}+x_{1} \bar{x}_{2}\right),
\end{gathered}
$$

where

$$
M(z)=\left(\begin{array}{cc}
-\sigma & \sigma \\
r-z & -a
\end{array}\right)
$$

which exhibit chaotic behavior when $\sigma=2, b=0.8$, $r=60+0.02 j$, and $a=1-0.06 j$. And the noise strength is $\sigma_{i}(y(t), y(t-\tau))=\mid 0.001 \sum_{k=1}^{N} a_{i k}\left(y_{k}(t)-y_{i}(t)\right)+$ $0.001 \sum_{k=1}^{N} b_{i k}\left(y_{k}(t-\tau)-y_{i}(t-\tau)\right) \mid$.

Consider complex projective synchronization in a driveresponse network coupled with $1+9$ identical complex Lorenz systems via impulsive pinning control, where the outer coupling matrices $A$ and $B$ are in Figures 1(a) and 1(b), respectively.

Choosing $\alpha=1+0.1 j, \Gamma=\operatorname{diag}(1,1), T=0.1$, and $\epsilon_{i k}=0.75$. By simple calculation, then one can choose $\varepsilon=200$ such that condition (18) holds. The initial values of complex state variables $y_{i}(t)(i=1,2, \ldots, 10)$ are chosen as $y_{i 1}(0)=$ $3+2 i+j(4+i)$ and $y_{i 2}(0)=(5+2 i)+j(6+2 i), x_{1}(0)=$ $2.040+2.020 j, x_{2}(0)=5.062+4.067 j, z=5.1$ is the synchronization orbit. Figures 2 and 3 show the evolution of synchronization trajectory and errors by impulsive pinning control, respectively.

\section{Conclusion}

The synchronization in drive-response stochastic coupled networks with complex-variable systems is considered in this paper. Because drive-response systems may evolve in different directions with a constant intersection angle in many real situations but may not simultaneously evolve along the same or inverse direction based on real number, real matrix, or even real function in a complex plane, we focus on the projective synchronization of this situation by impulsive pinning control through a theorem and a corollary. Eventually, several numerical simulations verified the validity of those results.

\section{Conflict of Interests}

The authors declare that there is no conflict of interests regarding the publication of this paper.

\section{Acknowledgments}

The authors thank the referees and the editor for their valuable comments on this paper. This work was supported by Shenzhen Strategic Emerging Industries Projects (ZDSY20120613125016389) and Shenzhen Basic Research Project (JCYJ20130331152625792).

\section{References}

[1] M. E. J. Newman, "The structure and function of complex networks," SIAM Review, vol. 45, no. 2, pp. 167-256, 2003.

[2] G. Shinar and M. Feinberg, "Structural sources of robustness in biochemical reaction networks," Science, vol. 327, no. 5971, pp. 1389-1391, 2010.

[3] A.-L. Barabási, "Scale-free networks: a decade and beyond," Science, vol. 325, no. 5939, pp. 412-413, 2009.

[4] C. T. Butts, "Revisiting the foundations of network analysis," Science, vol. 325, no. 5939, pp. 414-416, 2009.

[5] D. Centola, "The spread of behavior in an online social network experiment," Science, vol. 329, no. 5996, pp. 1194-1197, 2010.

[6] J.-W. Wang, Q. H. Ma, L. Zeng, and M. S. Abd-Elouahab, "Mixed outer synchronization of coupled complex networks with timevarying coupling delay," Chaos, vol. 21, no. 1, Article ID 013121, 2011.

[7] Q. T. Gan, R. Xu, and X. B. Kang, "Synchronization of chaotic neural networks with mixed time delays," Communications in Nonlinear Science and Numerical Simulation, vol. 16, no. 2, pp. 966-974, 2011.

[8] Q. X. Zhu and J. D. Cao, "Adaptive synchronization under almost every initial data for stochastic neural networks with time-varying delays and distributed delays," Communications in Nonlinear Science and Numerical Simulation, vol. 16, no. 4, pp. 2139-2159, 2011.

[9] A. Prasad, J. Kurths, and R. Ramaswamy, "The effect of timedelay on anomalous phase synchronization," Physics Letters A, vol. 372, no. 40, pp. 6150-6154, 2008.

[10] D. V. Senthilkumar, M. Lakshmanan, and J. Kurths, "Transition from phase to generalized synchronization in time-delay systems," Chaos, vol. 18, no. 2, Article ID 023118, 2008.

[11] I. Kanter, M. Zigzag, A. Englert, F. Geissler, and W. Kinzel, "Synchronization of unidirectional time delay chaotic networks and the greatest common divisor," Europhysics letters, vol. 93, no. 6, Article ID 60003, 2011.

[12] C.-K. Zhang, Y. He, and M. Wu, "Exponential synchronization of neural networks with time-varying mixed delays and sampled-data," Neurocomputing, vol. 74, no. 1-3, pp. 265-273, 2010.

[13] K. S. Sudheer and M. Sabir, "Adaptive modified function projective synchronization of multiple time-delayed chaotic Rössler system," Physics Letters A, vol. 375, no. 8, pp. 1176-1178, 2011.

[14] C. K. Ahn, "Anti-synchronization of time-delayed chaotic neural networks based on adaptive control," International Journal of Theoretical Physics, vol. 48, no. 12, pp. 3498-3509, 2009. 
[15] Z.-M. Ge, Y.-T. Wong, and S.-Y. Li, “Temporary lag and anticipated synchronization and anti-synchronization of uncoupled time-delayed chaotic systems," Journal of Sound and Vibration, vol. 318, no. 1-2, pp. 267-278, 2008.

[16] C. K. Ahn, "Adaptive $H_{\infty}$ anti-synchronization for time-delayed chaotic neural networks," Progress of Theoretical Physics, vol. 122, no. 6, pp. 1391-1403, 2009.

[17] D. Zhang and J. A. Xu, "Projective synchronization of different chaotic time-delayed neural networks based on integral sliding mode controller," Applied Mathematics and Computation, vol. 217, no. 1, pp. 164-174, 2010.

[18] Z. Y. Wu, J. Duan, and X. C. Fu, "Complex projective synchronization in coupled chaotic complex dynamical systems," Nonlinear Dynamics, vol. 69, no. 3, pp. 771-779, 2012.

[19] H. Y. Du, P. Shi, and N. Lü, "Function projective synchronization in complex dynamical networks with time delay via hybrid feedback control," Nonlinear Analysis: Real World Applications, vol. 14, no. 2, pp. 1182-1190, 2013.

[20] J.-G. Liu, "Generalized projective synchronization of fractionalorder complex networks with nonidentical nodes," Chinese Physics B, vol. 21, no. 12, Article ID 120506, 2012.

[21] H.-X. Yao and S.-G. Wang, "Cluster projective synchronization of complex networks with nonidentical dynamical nodes," Chinese Physics B, vol. 21, no. 11, Article ID 110506, 2012.

[22] Z. Y. Wu and X. C. Fu, "Complex projective synchronization in drive-response networks coupled with complex-variable chaotic systems," Nonlinear Dynamics, vol. 72, no. 1-2, pp. 9-15, 2013.

[23] Q. T. Gan, R. Xu, and X. B. Kang, "Synchronization of chaotic neural networks with mixed time delays," Communications in Nonlinear Science and Numerical Simulation, vol. 16, no. 2, pp. 966-974, 2011.

[24] X. J. Wu and H. T. Lu, "Generalized projective synchronization between two different general complex dynamical networks with delayed coupling," Physics Letters A, vol. 374, no. 38, pp. 3932-3941, 2010.

[25] D. Ghosh, "Generalized projective synchronization in timedelayed systems: nonlinear observer approach," Chaos, vol. 19, no. 1, Article ID 013102, 2009.

[26] D. Ghosh, S. Banerjee, and A. R. Chowdhury, "Generalized and projective synchronization in modulated time-delayed systems," Physics Letters A, vol. 374, no. 21, pp. 2143-2149, 2010.

[27] D. Ghosh, P. Saha, and A. R. Chowdhury, "Linear observer based projective synchronization in delay Rössler system," Communications in Nonlinear Science and Numerical Simulation, vol. 15, no. 6, pp. 1640-1647, 2010.

[28] J. R. Chen, L. C. Jiao, J. S. Wu, and X. D. Wang, "Projective synchronization with different scale factors in a drivenresponse complex network and its application in image encryption," Nonlinear Analysis: Real World Applications, vol. 11, no. 4, pp. 3045-3058, 2010.

[29] W. Wu, W. Zhou, and T. P. Chen, "Cluster synchronization of linearly coupled complex networks under pinning control," IEEE Transactions on Circuits and Systems, vol. 56, no. 4, pp. 829-839, 2009.

[30] C. Yuan and X. Mao, "Robust stability and controllability of stochastic differential delay equations with Markovian switching," Automatica, vol. 40, no. 3, pp. 343-354, 2004.

[31] S. Cai, J. Zhou, L. Xiang, and Z. Liu, "Robust impulsive synchronization of complex delayed dynamical networks," Physics Letters A, vol. 372, no. 30, pp. 4990-4995, 2008.
[32] Y. Yang and J. Cao, "Exponential synchronization of the complex dynamical networks with a coupling delay and impulsive effects," Nonlinear Analysis: Real World Applications, vol. 11, no. 3, pp. 1650-1659, 2010.

[33] W. Zhu, D. Xu, and Y. Huang, "Global impulsive exponential synchronization of time-delayed coupled chaotic systems," Chaos, Solitons \& Fractals, vol. 35, no. 5, pp. 904-912, 2008.

[34] C. Xu, J. Wang, and J. Feng, "Impulsive pinning Markovian switching stochastic complex networks with time-varying delay," Mathematical Problems in Engineering, vol. 2013, Article ID 461924, 9 pages, 2013.

[35] X. W. Liu and T. P. Chen, "Exponential synchronization of nonlinear coupled dynamical networks with a delayed coupling," Physica A, vol. 381, no. 1-2, pp. 82-92, 2007.

[36] C. Perret, The stability of numerical simulations of complex stochastic differential equations [Ph.D. thesis], ETH Zurich, Zurich, Switzerland, 2010. 


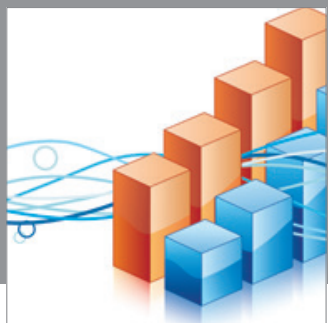

Advances in

Operations Research

mansans

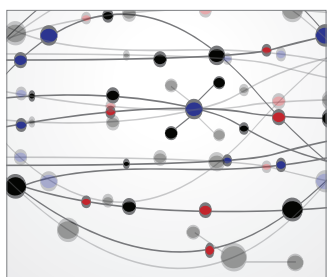

The Scientific World Journal
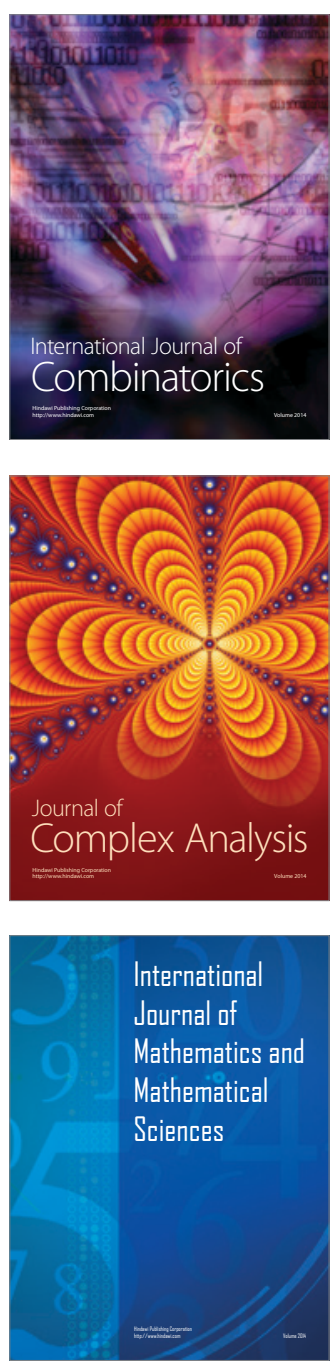
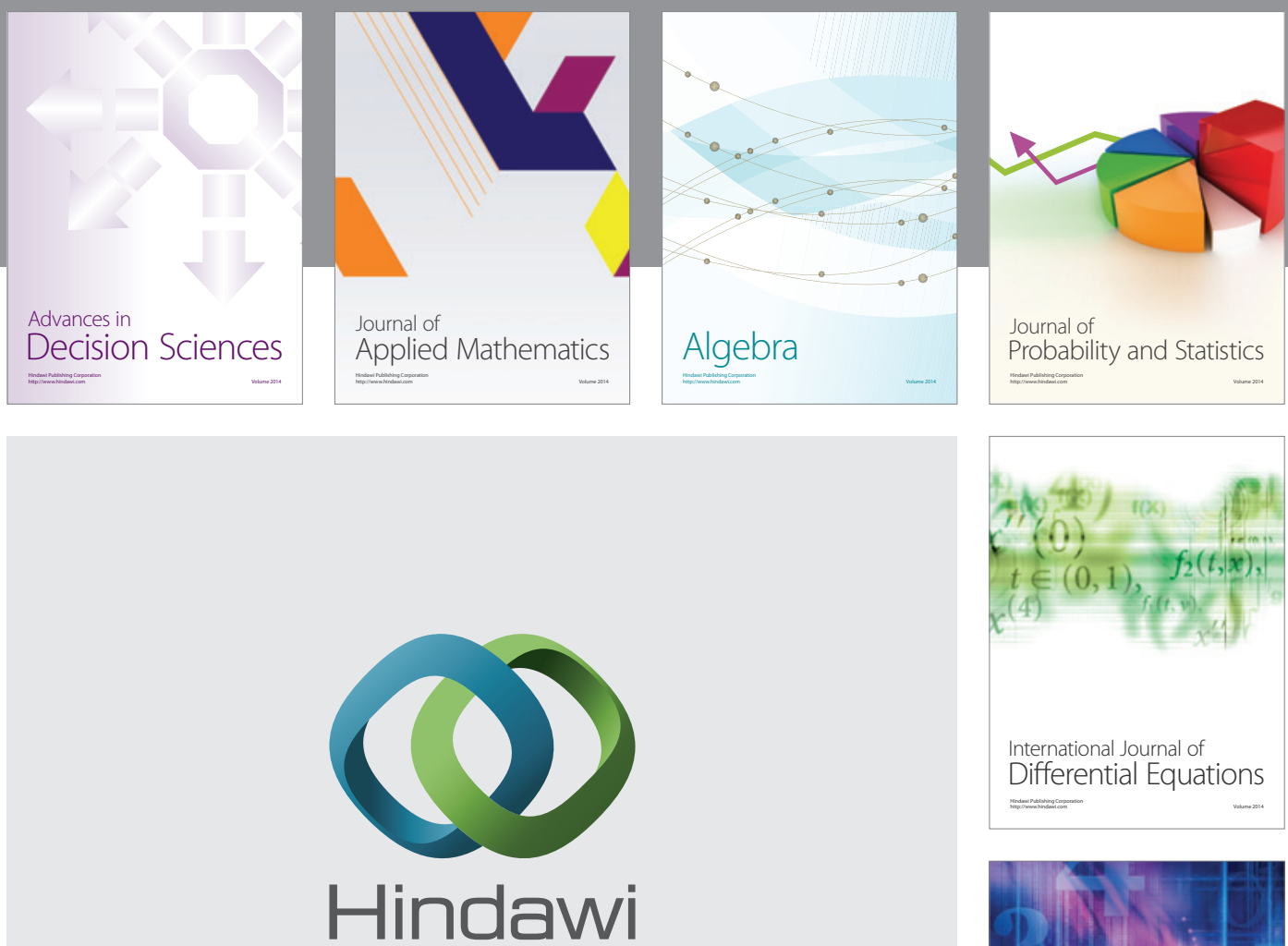

Submit your manuscripts at http://www.hindawi.com
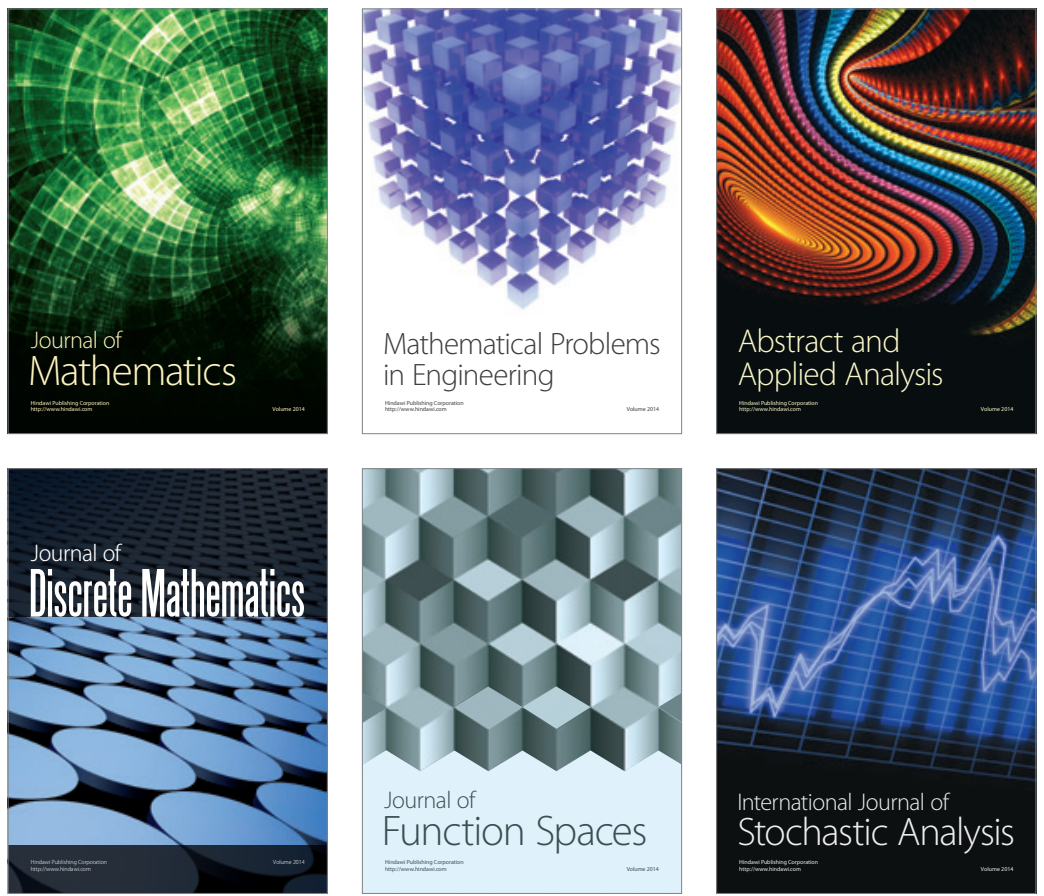

Journal of

Function Spaces

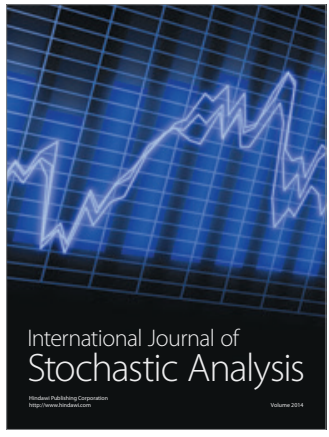

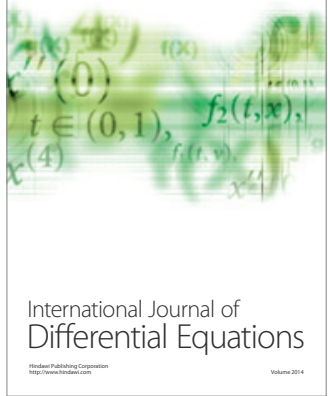
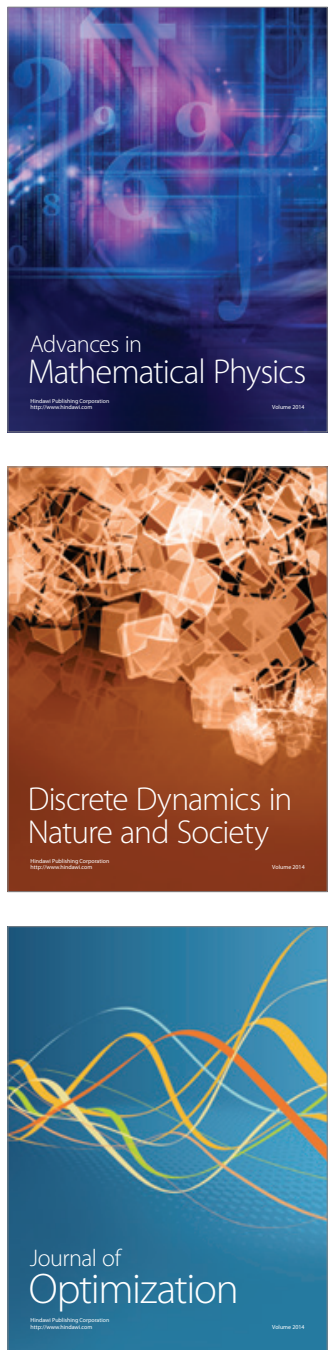